\title{
Instrumentos de avaliação para o diagnóstico da fraqueza muscular adquirida na unidade de terapia intensiva: Revisão narrativa
}

Assessment tools instruments for diagnostics of the intensive care unit acquired weakness:

\author{
Narrative review
}

Instrumentos de evaluación para el diagnóstico de la debilidad muscular adquirida en las unidades de cuidados intensivos: Revisión narrativa

Luciana Carrascal de Almeida ORCID: https://orcid.org/0000-0002-6083-0589 Instituto Educacional Campos, Brasil

E-mail: lucianacarrascal@hotmail.com Mayara Rodrigues Ribeiro Pereira ORCID: https://orcid.org/0000-0002-4111-9222 Instituto Educacional Campos, Brasil E-mail: mayara.rr21@hotmail.com

Jessica Delamuta Vitti ORCID: https://orcid.org/0000-0003-1987-3891 Instituto Educacional Campos, Brasil E-mail: jehvitti@hotmail.com

Nelson Francisco Serrão Júnior ORCID: https://orcid.org/0000-0002-0280-0752 Instituto Educacional Campos, Brasil

E-mail: nelsonserrao@unipampa.edu.br

\begin{abstract}
Resumo
Introdução: a fraqueza muscular adquirida (FMA) é uma condição corriqueira em pacientes de unidade de terapia intensiva (UTI) devido a inatividade, ventilação mecânica, uso de bloqueador neuromuscular, choque, sepse, insuficiência renal e hiperglicemia. A FMA é identificada por redução de força muscular generalizada, que atinge de forma simétrica músculos periféricos e respiratórios. Objetivo: verificar os principais instrumentos de avaliação para o diagnóstico de FMA na UTI e tratamentos disponíveis. Metodologia: trata-se de uma revisão de literatura, do tipo narrativa, realizada através de pesquisas nas bases de dados Lilacs, Medline e Pubmed, com estudos entre os anos de 2016 à 2020. Resultados: foram identificados 887 artigos inicialmente, dos quais foram excluídos 838 artigos a partir da leitura dos títulos e resumos, sendo que 49 destes foram lidos integralmente e 15 atendiam aos critérios de inclusão onde 8 tratavam sobre o diagnóstico e 7 a respeito do tratamento da FMA na UTI, os quais foram incluídos nesta revisão. Conclusão: a FMA na UTI é vivenciada frequentemente em pacientes críticos mantidos em repouso, os instrumentos de avaliação mais utilizados foram o Medical Research Council (MRC), a dinamometria e a ultrassonografia. A mobilização precoce é considerada como ferramenta para melhora funcional do doente crítico, sendo assim uma escolha viável e segura de tratamento.
\end{abstract}

Palavras-chave: Debilidade muscular; Unidades de Terapia Intensiva; Diagnóstico; Reabilitação; Terapêutica.

\begin{abstract}
Introduction: the intensive care unit acquired weakness (ICUAW) is a common condition in patients of the intensive care unit (ICU) due to inactivity, mechanical ventilation, use of neuromuscular blocker, chock, sepsis, renal failure and hyperglycemia. The ICUAW is identified by the overall reduction of the muscle strength, this affects in symmetrical ways the peripheral and respiratory muscle. Objective: verify the principals evaluation tools for the diagnostic of the ICUAW and available treatments. Methodology: review of narrative literature, through researches in the data base of Lilacs, Medline y PubMed, with studies between the years of 2016 until 2020. Results: originally were identified 8887 papers, where 838 were excluded by the reading of the summary and title, 49 papers were read in full and 15 more that attempted the criteria were included in this review, 8 treated about the diagnosis and 7 in respect of the ICUAW treatment in the ICU. Conclusion: the ICUAW is often experienced in critical patients kept in rest, the most used tolls for the diagnostic were the Medical Research Council (MRC), dynamometer and the ultrasound examination. The early mobilization is considered an improvement tool for the recovery of the critical sick, therefore is a secure and viable option for the treatment.
\end{abstract}

Keywords: Muscle Weakness; Intensive Care Units; Diagnosis; Rehabilitation; Therapeutics. 


\section{Resumen}

Introducción: la debilidad muscular adquirida es una condición corriente en pacientes de la unidad de cuidados intensivos (UCI), debido la inactividad, ventilación mecánica, uso de bloqueador neuromuscular, choque, sepsis, insuficiencia renal e hiperglucemia. La debilidad es identificada por la reducción de fuerza muscular generalizada, que atinge de modo simétrico músculos periféricos y respiratorios. Objetivo: verificar los principales instrumentos de evaluación para o diagnostico de la debilidad en la UCI e tratamientos disponibles. Metodología: revisión de literatura narrativa, mediante pesquisas en las bases de dados Lilacs, Medline y Pubmed, con estudios entre los años de 2016 hasta 2020. Resultados: se identificaron 887 artículos inicialmente, donde se excluyeron 838 artículos por la lectura dos títulos y resumes, 49 estudios han sido leídos integralmente e incluidos 15 en esta revisión que atendían a los criterios de inclusión donde hablaban sobre lo diagnostico o tratamiento de la debilidad muscular adquirida en la UCI,8 trataban del diagnóstico y 7 trataban sobre el tratamiento de la debilidad muscular adquirida en la UCI. Conclusión: la Debilidad en la UCI es vivenciada frecuentemente en pacientes críticos mantenidos en reposo, los instrumentos más utilizados para ese diagnostico fueran el Medical Research Council (MRC), la dinamometría y el examen de ultrasonido. La movilización precoz es considerada como herramienta para mejora funcional del enfermo critico, por tanto, una opción viable y segura de tratamiento.

Palabras clave: Debilidad Muscular; Unidades de Cuidados Intensivos; Diagnóstico; Rehabilitación; Terapéutica.

\section{Introdução}

O desenvolvimento de novas tecnologias para tratamento de pacientes internados em unidade de terapia intensiva (UTI), vem permitindo a diminuição da mortalidade de pacientes críticos (Aguiar, Westphal, Dadam, Mota, Pfutzenreuter \& França, 2019). A fraqueza muscular adquirida (FMA) é uma condição comum encontrada em pacientes em UTI devido a inatividade, a inflamação e o uso de agentes farmacológicos. Sendo definida por diminuição de força muscular generalizada, de forma simétrica, atingindo músculos periféricos e respiratórios, desenvolvida durante a internação na UTI sem que haja outra causa conhecida além da doença aguda (Doiron, Hoffmann \& Beller, 2018; Ballve, Dargains, Inchaustegui, Bratos, Percaz \& Ardariz et al., 2017).

Quanto à incidência a FMA é observada em 25 a $50 \%$ dos pacientes que permanecem mais que 5 dias em Ventilação Mecânica Invasiva (VMI), podendo prolongar tempo de internação, associado ao aumento da morbidade e mortalidade (Vicente, Amoza, García, Tocalini, Prieto \& Savio et al., 2020; Ballve et al., 2017). Dentre os sobreviventes 60 a $80 \%$ apresentarão comprometimento funcional (Zhou, Shi, Fan \& Zhu, 2020).

As causas deste acometimento são multifatoriais e incluem ventilação mecânica, repouso prolongado no leito, uso de bloqueador neuromuscular, choque, sepse, insuficiência renal e hiperglicemia (Ballve et al., 2017). A imobilidade resulta na atrofia muscular por desuso, promovendo a perda da massa muscular, afetando o sistema músculo esquelético nas alterações das fibras de miosina, promovido pelo estresse oxidativo, redução da síntese proteica e aumento da proteólise (Hodgson \& Tipping, 2017).

O diagnóstico exige que seja excluído qualquer outra causa de fraqueza muscular, além da doença crítica, recomendase que seja realizada uma avaliação de força muscular a beira leito utilizando o Medical Research Council (MRC), uma ferramenta simples e de fácil aplicabilidade, em que são testados 12 grupos musculares variando de 0 (ausência de contração) a 5 (força normal). A soma da pontuação varia de 0 a 60, e valores abaixo de 48 remetem ao diagnóstico de fraqueza muscular. Também pode ser utilizada como diagnóstico a dinamometria manual, sendo uma ferramenta interessante para avaliar a função neuromuscular, medindo a força muscular isométrica. Outro instrumento utilizado para identificar a FMA é a ultrassonografia muscular que identifica atrofia, alterações arquitetônicas e fasciculações (Hermans \& Van den Berghe, 2015; Latronico \& Gosselink, 2015).

Atuando na prevenção e tratamento da FMA vem se destacando a mobilização precoce, reduzindo o tempo de internação, melhorando a circulação, ventilação e capacidade física, além de ser uma técnica segura e efetiva (Castro-Avila, Serón, Fan, Gaete \& Mickan, 2015; Sachetti, Dal'Acqua, Lemos, Naue, Santos \& Bianchi et al., 2017; Zhou et al., 2020). 
Portanto, o objetivo deste estudo foi identificar os principais instrumentos de avaliação muscular para diagnóstico de FMA na UTI e verificar intervenções de tratamento, por meio de uma revisão narrativa da literatura.

\section{Metodologia}

O estudo consiste em uma revisão de literatura narrativa, com análise qualitativa dos artigos (Rother, 2007), que foi realizada nas bases de dados eletrônicas: Lilacs, Medline e Pubmed, limitada nas línguas inglesa, espanhola e portuguesa, com estudos realizados do ano de 2016 à 2020. Os descritores utilizados foram: “muscle weakness”, “intensive care units”, “diagnosis”, “rehabilitation”, "therapeutics" e suas variações em português e espanhol, através de pesquisa nos Descritores em Ciências da Saúde (DeCS).

Foram incluídos nesse estudo os ensaios clínicos e de coorte cujo desfecho era diagnóstico e/ou tratamento da FMA na UTI e que possuíssem o escore de STROBE acima de 11 pontos. Foram excluídos os artigos que não possuíam acesso na íntegra, que não incluíam o assunto pesquisado, estudos do tipo revisão de literatura, meta análise, estudos de caso e protocolos, artigos com publicação anterior ao ano de 2016, estudos cuja população foi composta por animais ou crianças, artigos duplicados e/ou que descrevessem os pacientes com FMA após a alta hospitalar à médio e longo prazo.

Além disso, foi utilizada uma adaptação da lista de verificação STROBE (von Elm, Altman, Egger, Pocock, Gøtzsche \& Vandenbroucke et al., 2008), uma ferramenta comumente usada para orientar o relato de estudos observacionais, a fim de avaliar a qualidade dos artigos incluídos no estudo. Os itens selecionados e adaptados para a presente revisão estão listados na Tabela 1. A adaptação da lista de verificação STROBE para a classificação da qualidade dos estudos incluídos nesta revisão narrativa consistiu-se de 15 itens, cada um representando um ponto ou meio ponto quando subdividido, desta forma, se o artigo pontuasse em todos os itens, recebia uma pontuação total de 15 pontos. Os pontos de corte estabelecidos para a classificação da qualidade foram: 0 a 5 pontos = baixa qualidade; 6 a 10 pontos = qualidade moderada; e 11 a 15 pontos = alta qualidade. Os 15 estudos que alcançaram qualidade alta, de acordo com os critérios mencionados acima, foram incluídos na análise final (Tabelas 2 e 3). 
Tabela 1. Adaptação da lista de verificação STROBE para classificação da qualidade dos estudos.

\begin{tabular}{|c|c|}
\hline Item & Tópico e recomendação \\
\hline 1 & $\begin{array}{l}\text { Título e Resumo: a) inclui no título e/ou resumo pelo menos uma das palavras-chave utilizadas na pesquisa; b) resumo } \\
\text { fornece uma sinopse do que foi realizado e encontrado. }\end{array}$ \\
\hline 2 & Introdução: explica os motivos e a base científica para a realização da pesquisa \\
\hline 3 & Introdução: inclui objetivos gerais e específicos, considerando claramente qualquer hipótese pré-estabelecida \\
\hline 4 & Métodos: apresenta no início do documento os principais elementos do desenho do estudo. \\
\hline 5 & $\begin{array}{l}\text { Métodos: descreve contexto, locais e datas relevantes, incluindo períodos de recrutamento, exposição, monitoramento } \\
\text { e coleta de dados. }\end{array}$ \\
\hline 6 & $\begin{array}{l}\text { Métodos: apresenta os critérios de elegibilidade, bem como as fontes e métodos de seleção dos participantes. } \\
\text { Especifica métodos de acompanhamento quando aplicável (estudos de coorte). }\end{array}$ \\
\hline 7 & $\begin{array}{l}\text { Métodos: define claramente todas as variáveis: resposta, exposição, predição, variáveis confundidoras e } \\
\text { modificadoras de efeito. Se aplicável, fornece critérios de diagnóstico. }\end{array}$ \\
\hline 8 & Métodos: especifica e detalha os métodos e instrumentos de medição. \\
\hline 9 & Métodos: o tamanho da amostra foi determinado racionalmente. \\
\hline 10 & $\begin{array}{l}\text { Métodos: a) explica como as variáveis quantitativas foram tratadas na análise; b) pelo menos um teste estatístico foi } \\
\text { realizado para verificar a relação em questão. }\end{array}$ \\
\hline 11 & $\begin{array}{l}\text { Resultados: a) apresenta pelo menos uma informação numérica relacionada à análise da associação em questão; b) se } \\
\text { as variáveis foram categorizadas, os pontos de corte foram descritos }\end{array}$ \\
\hline 12 & $\begin{array}{l}\text { Resultados: fornece estimativas não ajustadas e, se aplicável, ajustadas para fatores de confusão (especificar), bem } \\
\text { como sua precisão (por exemplo, intervalos de confiança de } 95 \% \text { ). }\end{array}$ \\
\hline 13 & Discussão: resume os principais resultados do estudo. \\
\hline 14 & Discussão: discute as limitações, considera possíveis fontes de viés ou imprecisão. \\
\hline 15 & $\begin{array}{l}\text { Discussão: a) apresenta uma interpretação geral prudente dos resultados, considerando objetivos, limitações, } \\
\text { multiplicidade de análises e resultados de estudos similares; b) discute a possibilidade de generalização dos resultados } \\
\text { (validade externa). }\end{array}$ \\
\hline
\end{tabular}

Fonte: von Elm et al. (2008).

A Tabela 1 apresenta uma adaptação da lista de verificação STROBE, que fornece uma pontuação aos artigos, sendo que o item 1 avalia o título e resumo, 2 e 3 a introdução, 4 ao 10 a metodologia, 11 e 12 os resultados e 13 ao 15 a discussão. Os estudos podem receber a pontuação de baixa, moderada e alta qualidade.

\section{Resultados}

Após uma busca criteriosa nas bases de dados foram identificados 887 artigos, em seguida foi realizada a leitura dos títulos e resumos dos quais foram excluídos 838 artigos (20 artigos em outros idiomas, 3 estudos de caso, 1 meta análise, 54 protocolos, 229 revisões, 8 estudos em população pediátrica, 5 estudos com animais, 312 que não falavam sobre o assunto, 45 que não abordavam o diagnóstico ou tratamento da FMA, 5 descreviam os pacientes com FMA após a alta hospitalar à médio e longo prazo, 137 artigos duplicados e 19 sem acesso do conteúdo na íntegra), sendo lidos integralmente 49 artigos, dos quais foram excluídos 34 estudos ( 2 estudos de caso, 13 tratavam de assunto diferente, 8 não abordavam sobre o diagnóstico ou tratamento da FMA, 3 eram sobre a FMA após a alta hospitalar à médio e longo prazo e 8 sem acesso ao conteúdo na íntegra), e portanto incluídos 15 nesta revisão, onde todos apresentaram alta qualidade através da avaliação STROBE e assim sendo nenhum precisou ser excluído. 
A Figura 1 apresenta as etapas metodológicas utilizadas para a seleção dos estudos. A tabela 2 e 3 descrevem a avaliação da qualidade dos estudos, que foram divididos em diagnóstico da FMA na UTI (tabela 2) e tratamento da FMA na UTI (tabela 3), ambas são uma adaptação da lista de verificação STROBE. Os artigos incluídos foram divididos em diagnóstico (Tabela 4) e tratamento (Tabela 5) da FMA.

Figura 1. Organograma da seleção dos artigos para o estudo.
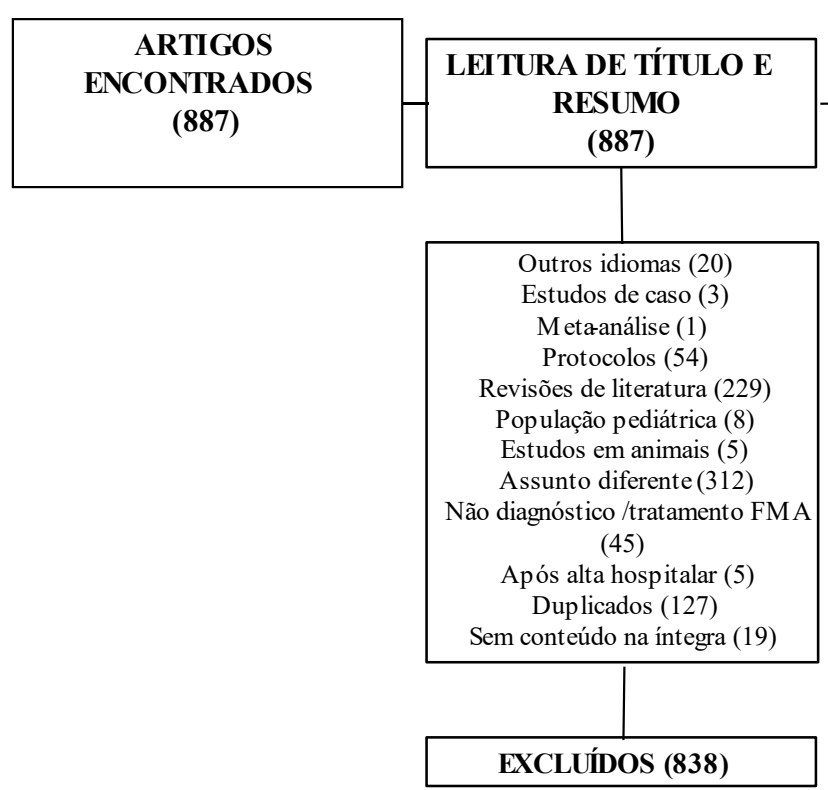
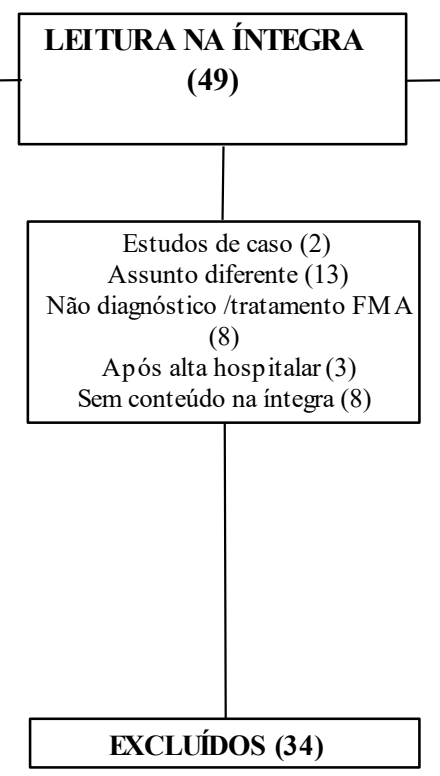

ARTIGOS INCLUÍDOS NA REVISÃO (15)

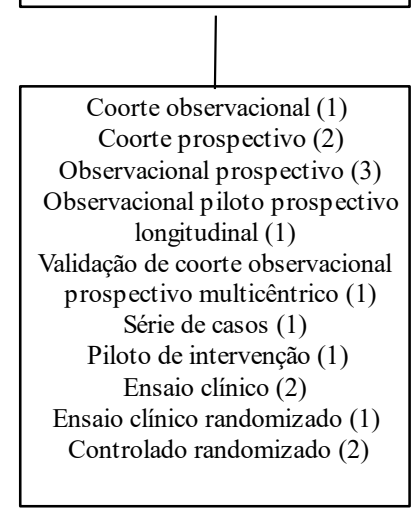

Ensaio clínico randomizado (1)

Controlado randomizado (2)

Fonte: Dados da pesquisa.

A Figura 1 expõe o organograma, discriminando o número de artigos encontrados, a quantidade de artigos excluídos, os critérios de exclusão e quantos artigos foram selecionados para esta revisão.

Tabela 2. Descrição da avaliação da qualidade dos estudos incluídos na tabela de diagnóstico de FMA. Critérios adaptados da lista de verificação STROBE.

\begin{tabular}{l|l|l|l|l|l|l|l|l|l|l|l|l|l|l|l|l}
\hline Artigo & 1 & 2 & 3 & 4 & 5 & 6 & 7 & 8 & 9 & 10 & 11 & 12 & 13 & 14 & 15 & Total \\
\hline Samosawala et al., (2016). & 1 & 1 & 1 & 1 & 1 & 1 & 0 & 1 & 1 & 1 & 1 & 1 & 1 & 1 & 0,5 & 13,5 \\
\hline Kawahara et al., (2016). & 1 & 1 & 1 & 1 & 1 & 1 & 1 & 1 & 1 & 1 & 1 & 1 & 1 & 1 & 0,5 & 14,5 \\
\hline Hernandéz-Socorro et al., (2018). & 1 & 1 & 1 & 1 & 1 & 1 & 1 & 1 & 1 & 1 & 1 & 1 & 1 & 0 & 0,5 & 13,5 \\
\hline Kelmenson et al., (2018). & 1 & 1 & 1 & 1 & 1 & 1 & 1 & 1 & 1 & 1 & 1 & 1 & 1 & 1 & 1 & 15 \\
\hline Patejdl et al., (2019). & 1 & 1 & 1 & 1 & 1 & 1 & 1 & 1 & 0 & 1 & 1 & 1 & 1 & 1 & 1 & 14 \\
\hline Bragança et al., (2019). & 1 & 1 & 1 & 1 & 1 & 1 & 1 & 1 & 1 & 1 & 1 & 1 & 1 & 1 & 1 & 15 \\
\hline Xie et al., (2020). & 0,5 & 1 & 1 & 1 & 1 & 1 & 1 & 1 & 1 & 1 & 1 & 1 & 1 & 1 & 1 & 14,5 \\
\hline Witteveen et al., (2020). & 1 & 1 & 1 & 1 & 1 & 1 & 1 & 1 & 1 & 1 & 1 & 1 & 1 & 1 & 1 & 15 \\
\hline
\end{tabular}

Fonte: Dados da pesquisa. 
Tabela 3. Descrição da avaliação da qualidade dos estudos incluídos na tabela de tratamento de FMA. Critérios adaptados da lista de verificação STROBE.

\begin{tabular}{l|l|l|l|l|l|l|l|l|l|l|l|l|l|l|l|l}
\hline Artigo & 1 & 2 & 3 & 4 & 5 & 6 & 7 & 8 & 9 & 10 & 11 & 12 & 13 & 14 & 15 & Total \\
\hline Enciso-Olivera et al., (2016). & 0,5 & 1 & 1 & 1 & 1 & 1 & 1 & 1 & 0 & 1 & 1 & 1 & 1 & 1 & 1 & 13,5 \\
\hline Wollersheim et al., (2017). & 1 & 1 & 1 & 1 & 1 & 1 & 1 & 1 & 0 & 1 & 1 & 1 & 1 & 1 & 1 & 14 \\
\hline Sommers et al., (2017). & 1 & 1 & 0 & 1 & 1 & 1 & 1 & 1 & 1 & 1 & 1 & 1 & 1 & 1 & 1 & 14 \\
\hline Sarfati et al., (2018). & 1 & 1 & 1 & 1 & 1 & 1 & 1 & 1 & 1 & 1 & 0,5 & 1 & 1 & 1 & 0,5 & 14 \\
\hline Veldema et al., (2019). & 1 & 1 & 1 & 1 & 1 & 1 & 1 & 1 & 1 & 1 & 1 & 1 & 1 & 0 & 1 & 14 \\
\hline Nakamura et al., (2019). & 1 & 1 & 1 & 1 & 1 & 1 & 1 & 1 & 1 & 1 & 1 & 1 & 1 & 1 & 1 & 15 \\
\hline França et al., (2020). & 1 & 1 & 1 & 1 & 1 & 1 & 1 & 1 & 1 & 1 & 1 & 1 & 1 & 1 & 1 & 15 \\
\hline
\end{tabular}

Fonte: Dados da pesquisa.

As Tabelas 2 e 3 apresentam a qualidade dos estudos sobre o diagnóstico (Tabela 2) e tratamento (Tabela 3) de FMA na UTI, através da lista de verificação STROBE, onde todos artigos apresentaram alta qualidade.

Tabela 4. Resultado dos artigos encontrados do diagnóstico de FMA.

\begin{tabular}{|c|c|c|c|c|}
\hline Autor/Ano & Tipo de estudo & Objetivo & Variáveis & Conclusão \\
\hline $\begin{array}{l}\text { Samosawala et al., } \\
\text { (2016). }\end{array}$ & Coorte observacional & $\begin{array}{l}\text { Observar mudanças nos } \\
\text { valores de dinamometria } \\
\text { para bíceps, tríceps e } \\
\text { deltoide na extremidade } \\
\text { superior e quadríceps, } \\
\text { dorsiflexores e flexores } \\
\text { plantares nas } \\
\text { extremidades inferiores } \\
\text { durante internação na } \\
\text { UTI. }\end{array}$ & $\begin{array}{l}\text { Foi utilizado o dinamômetro } \\
\text { portátil para mensurar a força } \\
\text { gerada para cada músculo nos dias } \\
1,3,5 \text { e } 7 \text {, os pacientes foram } \\
\text { instruídos para realizar contração } \\
\text { isométrica máxima durante a } \\
\text { medida, realizaram } 3 \text { esforços para } \\
\text { cada músculo e o melhor entre as } \\
3 \text { tentativas foi registrado. }\end{array}$ & $\begin{array}{l}\text { Esse estudo mostrou uma } \\
\text { redução progressiva na força } \\
\text { muscular periférica medida } \\
\text { pelo dinamômetro portátil } \\
\text { durante o período inicial de } \\
\text { internação na UTI. }\end{array}$ \\
\hline $\begin{array}{l}\text { Kawahara et al., } \\
(2016) .\end{array}$ & $\begin{array}{l}\text { Estudo observacional } \\
\text { prospectivo }\end{array}$ & $\begin{array}{l}\text { Avaliar o grau de atrofia } \\
\text { do músculo esquelético } \\
\text { durante uma internação } \\
\text { relativamente curta na } \\
\text { UTI, identificar a região } \\
\text { do músculo que é } \\
\text { suscetível ao desuso em } \\
\text { pacientes criticamente } \\
\text { enfermos e se o grau de } \\
\text { atrofia era diferente com o } \\
\text { nível de AVDs antes da } \\
\text { admissão. }\end{array}$ & $\begin{array}{l}\text { Pacientes que necessitaram de } \\
\text { repouso no leito na admissão da } \\
\text { UTI, foram divididos em } 2 \text { grupos: } \\
\text { grupo dependentes e } \\
\text { independentes nas AVDs antes da } \\
\text { admissão na UTI. O grau de } \\
\text { atrofia do músculo esquelético foi } \\
\text { avaliado no dia, } 72 \text { e } 144 \text { horas } \\
\text { após admissão na UTI, medindo } \\
\text { circunferência dos MMII e } \\
\text { MMSS. }\end{array}$ & $\begin{array}{l}\text { A atrofia muscular por desuso } \\
\text { em pacientes com doenças } \\
\text { graves desenvolveram-se } \\
\text { rapidamente, especialmente } \\
\text { nos MMII em pacientes que } \\
\text { eram independentes nas } \\
\text { AVDs antes da admissão na } \\
\text { UTI. Este resultado enfatiza a } \\
\text { necessidade de reabilitação } \\
\text { precoce intensiva } \\
\text { concentrando-se nos } \\
\text { músculos dos MMII para } \\
\text { prevenir a atrofia muscular. }\end{array}$ \\
\hline $\begin{array}{l}\text { Hernandéz-Socorro } \\
\text { et al., (2018). }\end{array}$ & $\begin{array}{l}\text { Observacional } \\
\text { prospectivo }\end{array}$ & $\begin{array}{l}\text { Investigar um novo } \\
\text { protocolo de } \\
\text { ultrassonografia, } \\
\text { confiável e estruturado } \\
\text { para avaliar a quantidade } \\
\text { e qualidade dos músculos } \\
\text { na sarcopenia em } \\
\text { pacientes com suspeita de } \\
\text { fraqueza muscular } \\
\text { adquirida na UTI. }\end{array}$ & $\begin{array}{l}\text { Foi realizada uma análise } \\
\text { multivariada em } 29 \text { casos e } 19 \\
\text { controles, utilizando um protocolo } \\
\text { de US que avalia a qualidade do } \\
\text { músculo QRF em tempo real } \\
\text { modo B, Doppler colorido e US } \\
\text { em modo M e avaliar a espessura } \\
\text { do tendão central intramuscular } \\
\text { QRF, a área de secção transversal } \\
\text { e a espessura do músculo no modo } \\
\text { B de US. }\end{array}$ & $\begin{array}{l}\text { Um protocolo de US permitiu } \\
\text { avaliar as mudanças } \\
\text { quantitativas e qualitativas no } \\
\text { músculo QRF em pacientes } \\
\text { graves, ventilados } \\
\text { mecanicamente com suspeita } \\
\text { clínica de fraqueza } \\
\text { neuromuscular } \\
\text { adquirida. Entre as alterações } \\
\text { quantitativas do US, a área e a } \\
\text { espessura muscular } \\
\text { diminuíram a a } \\
\text { significativamente e a } \\
\text { espessura do tendão central do }\end{array}$ \\
\hline
\end{tabular}




\begin{tabular}{|c|c|c|c|c|}
\hline & & & & $\begin{array}{l}\text { QRF aumentou } \\
\text { significativamente nos casos } \\
\text { em relação aos } \\
\text { controles. Esses achados são } \\
\text { relevantes porque mostram, } \\
\text { pela primeira vez, que o } \\
\text { tendão central QRF não sofre } \\
\text { atrofia devido à sua natureza } \\
\text { de tecido conjuntivo } \\
\text { denso, isto foi } \\
\text { ultrassonograficamente mais } \\
\text { evidente em pacientes graves } \\
\text { catabolicamente com perda de } \\
\text { músculo QRF. }\end{array}$ \\
\hline $\begin{array}{l}\text { Kelmenson et al., } \\
\text { (2018). }\end{array}$ & Coorte prospectivo & $\begin{array}{l}\text { Avaliar a generalização e } \\
\text { validade dos estudos de } \\
\text { condução nervosa única e } \\
\text { testes de US muscular } \\
\text { para identificar } \\
\text { polineuropatia de doença } \\
\text { crítica e também a } \\
\text { capacidade do US } \\
\text { muscular para avaliar o } \\
\text { prognóstico } \\
\text { pacientes. }\end{array}$ & $\begin{array}{l}\text { Pacientes com permanência na } \\
\text { UTI por mais de } 48 \text { horas } \\
\text { requerendo VM, foram avaliados } \\
\text { semanalmente pelo MRC, teste de } \\
\text { condução nervosa única, US e } \\
\text { eletromiografia. }\end{array}$ & $\begin{array}{l}\text { A condução nervosa única e o } \\
\text { US muscular alcançaram } \\
\text { acurácia diagnóstica para } \\
\text { pacientes em risco de } \\
\text { polineuropatia de doença } \\
\text { crítica. A utilização desses } \\
\text { testes pode ser benéfica para } \\
\text { pacientes criticamente } \\
\text { enfermos com risco de } \\
\text { polineuropatia de doença } \\
\text { crítica. }\end{array}$ \\
\hline Patejdl et al., (2019). & $\begin{array}{l}\text { Observacional piloto } \\
\text { prospectivo } \\
\text { longitudinal }\end{array}$ & $\begin{array}{l}\text { Investigar a relação entre } \\
\text { o aumento da } \\
\text { ecogenecidade muscular, } \\
\text { marcadores séricos de } \\
\text { inflamação, dano } \\
\text { endotelial e sua relevância } \\
\text { para fraqueza adquirida } \\
\text { na UTI em pacientes } \\
\text { criticamente enfermos. }\end{array}$ & $\begin{array}{l}\text { No estudo foi utilizado dois } \\
\text { grupos: grupo composto por } \\
\text { indivíduos saudáveis e grupo de } \\
\text { pacientes de UTI. } \\
\text { Foram avaliados sinais de } \\
\text { fraqueza muscular utilizando } \\
\text { MRC, a ecogenecidade com } \\
\text { ultrassonografia nos músculos: } \\
\text { quadríceps femoral, tibial anterior, } \\
\text { bíceps braquial e braquiorradial } \\
\text { bilateral; além de marcadores } \\
\text { séricos de inflamação Syndecan-1 } \\
\text { nos dias } 3 \text { e } 10 .\end{array}$ & $\begin{array}{l}\text { Avaliações puramente clínica } \\
\text { e eletrofísicas de pacientes na } \\
\text { UTI são insuficientes para } \\
\text { diagnóstico de fraqueza } \\
\text { adquirida na UTI nos } \\
\text { primeiros cursos da doença. } \\
\text { O uso combinado de } \\
\text { varreduras de ultrassom de } \\
\text { músculos e biomarcadores } \\
\text { inflamatórios pode, portanto, } \\
\text { ser útil no diagnóstico da } \\
\text { síndrome de fraqueza } \\
\text { adquirida em terapia intensiva } \\
\text { e na previsão do resultado de } \\
\text { longo prazo para a saúde de } \\
\text { pacientes com doenças } \\
\text { graves. }\end{array}$ \\
\hline $\begin{array}{l}\text { Bragança et al., } \\
(2019) .\end{array}$ & Coorte prospectivo & $\begin{array}{l}\text { Testar a concordância } \\
\text { entre a dinamometria de } \\
\text { preensão manual e o } \\
\text { MRC para o diagnóstico } \\
\text { de fraqueza adquirida na } \\
\text { UTI e avaliar se os } \\
\text { achados de dinamometria } \\
\text { estão associados à } \\
\text { morbidade e mortalidade. }\end{array}$ & $\begin{array}{l}\text { Pacientes que precisaram de pelo } \\
\text { menos } 5 \text { dias de cuidados críticos } \\
\text { foram incluídos e avaliados pelo } \\
\text { MRC e dinamometria. }\end{array}$ & $\begin{array}{l}\text { A FMA na UTI está associada } \\
\text { a maior tempo de internação e } \\
\text { retardo no desmame da VM } \\
\text { durante o período de pelo } \\
\text { menos } 6 \text { meses, sugerindo que } \\
\text { esta condição está relacionada } \\
\text { a consequências de longo } \\
\text { prazo. A dinamometria de } \\
\text { preensão manual pode } \\
\text { fornecer uma alternativa ao } \\
\text { exame MRC, sendo um } \\
\text { método simples, rápido e } \\
\text { preciso para o diagnóstico de } \\
\text { FMA na UTI. }\end{array}$ \\
\hline Xie et al., (2020). & $\begin{array}{l}\text { Observacional } \\
\text { prospectivo }\end{array}$ & $\begin{array}{l}\text { Esclarecer a relação entre } \\
\text { GDF-15 e perda muscular } \\
\text { em pacientes de UTI } \\
\text { tratados com VM, bem } \\
\text { como avaliar sua utilidade }\end{array}$ & $\begin{array}{l}\text { Os pacientes foram divididos em } \\
\text { dois grupos: grupo fraqueza } \\
\text { muscular adquirida na UTI e } \\
\text { grupo sem fraqueza muscular } \\
\text { adquirida na UTI. }\end{array}$ & $\begin{array}{l}\text { A elevação sustentada do } \\
\text { nível de concentração } \\
\text { plasmática do GDF-15 foi } \\
\text { associada a perda de massa } \\
\text { muscular em pacientes }\end{array}$ \\
\hline
\end{tabular}




\begin{tabular}{|c|c|c|c|c|}
\hline & & $\begin{array}{l}\text { como biomarcador } \\
\text { precoce de perda } \\
\text { muscular no diagnóstico } \\
\text { precoce de FMA na UTI. }\end{array}$ & $\begin{array}{l}\text { Foram coletadas amostras de } \\
\text { sangue nos dias } 1 \text {, } 4 \text { e } 7 \text {, nesses } \\
\text { dias todos os analgésicos e } \\
\text { sedativos foram suspensos, o nível } \\
\text { plasmático de GDF-15 foi } \\
\text { detectado por ELISA, a área do } \\
\text { reto femoral foi medida por US e a } \\
\text { força muscular por MRC. }\end{array}$ & $\begin{array}{l}\text { internados na UTI. O nível de } \\
\text { plasma GDF-15 no dia } 7 \text { tem } \\
\text { um alto rendimento } \\
\text { diagnóstico para fraqueza } \\
\text { muscular adquirida na UTI, } \\
\text { demonstrou ser um candidato } \\
\text { a biomarcador ideal para } \\
\text { perda de massa muscular em } \\
\text { fraqueza muscular adquirida } \\
\text { na UTI. }\end{array}$ \\
\hline $\begin{array}{l}\text { Witteveen et al., } \\
(2020) .\end{array}$ & $\begin{array}{l}\text { Validação de coorte } \\
\text { observacional } \\
\text { prospectivo } \\
\text { multicêntrico }\end{array}$ & $\begin{array}{l}\text { Validar externamente e se } \\
\text { necessário atualizar o } \\
\text { modelo de predição } \\
\text { desenvolvido } \\
\text { anteriormente para FMA } \\
\text { na UTI. A validação } \\
\text { externa incluiu validação } \\
\text { temporal (pacientes de um } \\
\text { período posterior) e } \\
\text { geográfica (pacientes de } \\
\text { outras instituições) para } \\
\text { avaliar a generalização do } \\
\text { modelo. }\end{array}$ & $\begin{array}{l}\text { Pacientes recém admitidos na UTI } \\
\text { e em VM em } 48 \text { horas foram } \\
\text { avaliados com a relação } \\
\text { PaO2/FiO2, transfusão de } \\
\text { eritrócitos, hipercalcemia e } \\
\text { hipofosfatemia, MRC quando } \\
\text { pacientes estavam alertas, } \\
\text { APACHE IV e SOFA. }\end{array}$ & $\begin{array}{l}\text { O modelo de predição } \\
\text { desenvolvido anteriormente } \\
\text { para FMA na UTI mostrou } \\
\text { baixo desempenho em uma } \\
\text { nova coorte de validação } \\
\text { multicêntrica independente. } \\
\text { Os métodos de atualização do } \\
\text { modelo melhoraram a } \\
\text { calibração, mas não a } \\
\text { discriminação. O modelo de } \\
\text { previsão recém derivado } \\
\text { mostrou discriminação justa e } \\
\text { classificou os pacientes } \\
\text { melhor do que os escores } \\
\text { APACHE IV e SOFA. Isso } \\
\text { indica que a previsão precoce } \\
\text { de FMA na UTI ainda é um } \\
\text { desafio e precisa de mais } \\
\text { atenção. }\end{array}$ \\
\hline
\end{tabular}

UTI: Unidade de terapia intensiva; AVD's: Atividades de vida diária; MMII: Membros inferiores; MMSS: Membros superiores; VM: Ventilação mecânica; US: Ultrassom; QRF: Quadríceps reto femoral; MRC: Medical research council; FMA: Fraqueza muscular adquirida; GDF-15: Grouth differentiatton factor-15; ELISA: Enzyme-linked immunosorbent assay; PaO2: Pressão parcial de oxigênio no sangue arterial; FiO2: Fração inspirada de oxigênio; APACHE IV: Acute physiology and chronic health evaluation IV; SOFA: Sequential organ failure assessment. Fonte: Dados da pesquisa.

Tabela 5. Resultado dos artigos encontrados do tratamento de FMA.

\begin{tabular}{|c|c|c|c|c|}
\hline Autor/Ano & Tipo de estudo & Objetivo & Variáveis & Conclusão \\
\hline $\begin{array}{l}\text { Enciso-Olivera et al., } \\
(2016) .\end{array}$ & Série de casos & $\begin{array}{l}\text { Descrever a resposta } \\
\text { cardiovascular em paciente } \\
\text { internados em UTI aplicando } \\
\text { um protocolo de atividade física } \\
\text { direcionada como parte da } \\
\text { reabilitação, a fim de estabelecer } \\
\text { as bases para o desenvolvimento } \\
\text { de um modelo de intervenção } \\
\text { em pacientes críticos. }\end{array}$ & $\begin{array}{l}\text { Pacientes que tiveram permanência } \\
\text { na unidade igual ou superior a } 72 \\
\text { horas, que apresentavam requisitos de } \\
\text { VM foram avaliados pelo MRC e } \\
\text { escala de Borg, frequência cardíaca, } \\
\text { pressão arterial sistólica e diastólica. } \\
\text { Foram avaliados antes, durante e } \\
\text { depois da sessão de exercícios com } \\
\text { cargas aplicadas ajustadas por um } \\
\text { especialista. }\end{array}$ & $\begin{array}{l}\text { Em pacientes críticos o uso } \\
\text { de cargas direcionadas } \\
\text { pelo especialista não tem } \\
\text { efeito cardiovascular } \\
\text { significativo e pode ser } \\
\text { mantido como parte dos } \\
\text { protocolos de reabilitação. }\end{array}$ \\
\hline
\end{tabular}


Research, Society and Development, v. 10, n. 8, e12010817077, 2021

(CC BY 4.0) | ISSN 2525-3409 | DOI: http://dx.doi.org/10.33448/rsd-v10i8.17077

\begin{tabular}{|c|c|c|c|c|}
\hline & & & & $\begin{array}{l}\text { muscular e, portanto, pode } \\
\text { ter potencial de prevenir e } \\
\text { tratar a FMA na UTI. }\end{array}$ \\
\hline $\begin{array}{l}\text { Sommers et al., } \\
\text { (2017). }\end{array}$ & Ensaio clínico & $\begin{array}{l}\text { Explorar a viabilidade do } \\
\text { treinamento em esteira com } \\
\text { suporte de peso corporal em } \\
\text { pacientes com condições críticas } \\
\text { na UTI. }\end{array}$ & $\begin{array}{l}\text { Pacientes admitidos na UTI e em VM } \\
\text { por mais de } 48 \text { horas, foi realizado } \\
\text { esteira com suporte de peso à beira do } \\
\text { leito. }\end{array}$ & $\begin{array}{l}\text { Este estudo demonstrou } \\
\text { que o treinamento em } \\
\text { esteira com suporte de } \\
\text { peso corporal é seguro, } \\
\text { viável, reduz mão de obra } \\
\text { e facilita a primeira vez } \\
\text { para deambulação em } \\
\text { pacientes críticos com } \\
\text { fraqueza muscular severa } \\
\text { na UTI. }\end{array}$ \\
\hline Sarfati et al., (2018). & $\begin{array}{l}\text { Controlado } \\
\text { randomizado }\end{array}$ & $\begin{array}{l}\text { Investigar se a inclinação } \\
\text { passiva adicionada a uma } \\
\text { terapia de reabilitação } \\
\text { padronizada melhora a força na } \\
\text { alta da UTI em pacientes após } \\
\text { cirurgia cardiotorácica. }\end{array}$ & $\begin{array}{l}\text { Pacientes em VM por } 3 \text { dias ou mais } \\
\text { foram divididos em } 2 \text { grupos: grupo } \\
\text { terapia de reabilitação padronizada e } \\
\text { inclinação e grupo sem inclinação } \\
\text { (somente reabilitação). }\end{array}$ & $\begin{array}{l}\text { A inclinação passiva } \\
\text { adicionada a uma terapia } \\
\text { de reabilitação } \\
\text { padronizada não melhorou } \\
\text { força muscular na alta da } \\
\text { UTI em pacientes } \\
\text { cirúrgicos, mesmo que } \\
\text { uma recuperação mais } \\
\text { rápida com inclinação } \\
\text { tenho sido sugerida. }\end{array}$ \\
\hline $\begin{array}{l}\text { Veldema et al., } \\
\text { (2019). }\end{array}$ & $\begin{array}{l}\text { Ensaio clínico } \\
\text { controlado } \\
\text { randomizado }\end{array}$ & $\begin{array}{l}\text { Investigar o efeito do } \\
\text { treinamento do cicloergômetro e } \\
\text { do treinamento de resistência } \\
\text { aplicado por cinco dias por } \\
\text { semana durante um período de } \\
\text { quatro semanas, além de } \\
\text { cuidados padrão durante } \\
\text { reabilitação de pacientes } \\
\text { internados com FMA na UTI. }\end{array}$ & $\begin{array}{l}\text { Pacientes internados na UTI foram } \\
\text { divididos em } 3 \text { grupos: } \\
\text { grupo treinamento ergométrico, } \\
\text { cicloergômetro em MMII durante } 20 \\
\text { minutos por } 5 \text { dias na semana; } \\
\text { grupo resistência, treinamento de } \\
\text { resistência muscular de MMII e } \\
\text { tronco durante } 20 \text { minutos, } 5 \text { dias na } \\
\text { semana e } \\
\text { grupo controle, recebeu apenas } \\
\text { cuidados padrão. }\end{array}$ & $\begin{array}{l}\text { Sugere-se que o } \\
\text { treinamento ergométrico } \\
\text { ou treinamento de } \\
\text { resistência pode aumentar } \\
\text { a eficácia do tratamento } \\
\text { padrão para melhorar a } \\
\text { força rascular, } \\
\text { capacidade de locomoção } \\
\text { e aptidão } \\
\text { cardiorrespiratória durante } \\
\text { reabilitação de pacientes } \\
\text { com fraqueza muscular } \\
\text { adquirida na UTI. }\end{array}$ \\
\hline $\begin{array}{l}\text { Nakamura et al., } \\
\text { (2019). }\end{array}$ & $\begin{array}{l}\text { Ensaio clínico } \\
\text { randomizado }\end{array}$ & $\begin{array}{l}\text { Avaliar a eficácia da } \\
\text { estimulação elétrica muscular } \\
\text { com eletrodo de cinta na } \\
\text { redução da perda do volume } \\
\text { muscular na fase inicial em } \\
\text { pacientes de UTI. }\end{array}$ & $\begin{array}{l}\text { Os pacientes foram divididos em } 2 \\
\text { grupos: } \\
\text { grupo estimulação elétrica muscular } \\
\text { que recebeu eletroestimulação com } \\
\text { eletrodo de cinta em quadríceps } \\
\text { femoral e } \\
\text { grupo controle que não recebeu a } \\
\text { intervenção. O volume do músculo } \\
\text { quadríceps femoral foi avaliado com } \\
\text { tomografia computadorizada nos dias } \\
1 \text { e } 10 \text { da intervenção. }\end{array}$ & $\begin{array}{l}\text { A estimulação elétrica } \\
\text { muscular pode ser } \\
\text { introduzida para pacientes } \\
\text { criticamente enfermos } \\
\text { durante a fase aguda de } \\
\text { terapia intensiva A } \\
\text { estimulação elétrica } \\
\text { muscular pode inibir } \\
\text { significativamente a taxa } \\
\text { de perda do volume } \\
\text { muscular. }\end{array}$ \\
\hline
\end{tabular}




\begin{tabular}{|c|c|c|c|c|}
\hline França et al., (2020). & Ensaio clínico & $\begin{array}{l}\text { Avaliar o efeito agudo da } \\
\text { cicloergometria passiva de } \\
\text { MMII e da estimulação elétrica } \\
\text { funcional em quadríceps sobre o } \\
\text { estresse nitrosativo e as } \\
\text { citocinas inflamatórias em } \\
\text { pacientes críticos. }\end{array}$ & $\begin{array}{l}\text { Pacientes sob ventilação mecânica } \\
\text { foram divididos em } 4 \text { grupos: } \\
\text { grupo controle, onde não receberam } \\
\text { intervenção; grupo cicloergometria } \\
\text { passiva, cicloergometria em MMII } \\
\text { durante } 20 \text { minutos; } \\
\text { grupo estimulação elétrica funcional, } \\
\text { estimulação elétrica em reto femoral } \\
\text { e vasto lateral bilateral durante } 20 \\
\text { minutos e grupo cicloergometria } \\
\text { passiva + estimulação elétrica } \\
\text { funcional ambas as intervenções } \\
\text { duraram } 20 \text { minutos cada em um total } \\
\text { de } 40 \text { minutos. }\end{array}$ & $\begin{array}{l}\text { Ambas intervenções não } \\
\text { alteraram os níveis séricos } \\
\text { de citocinas inflamatórias. } \\
\text { Porém avaliado os efeitos } \\
\text { destas terapias indicam } \\
\text { reduzir o estresse } \\
\text { nitrosativo quando usadas } \\
\text { separadamente. }\end{array}$ \\
\hline
\end{tabular}

UTI: Unidade de terapia intensiva; VM: Ventilação mecânica; MRC: Medical research council; FMA: Fraqueza muscular adquirida; MMII: membros inferiores. Fonte: Dados da pesquisa.

As Tabelas 4 e 5 apresentam o autor, ano, tipo de estudo, objetivo, variáveis analisadas e conclusão de cada estudo sobre o diagnóstico (Tabela 4) e tratamento (Tabela 5) de FMA na UTI, indicando as características de cada estudo incluído nesta revisão.

\section{Discussão}

A FMA que se desenvolve durante a internação na UTI, é uma complicação frequente de doença crítica e está associada a alta morbidade e mortalidade, podendo ter consequências a longo prazo, além da fase de hospitalização (Ballve et al., 2017; Vicente et al., 2020). Dessa forma o reconhecimento precoce das alterações musculares, além de ser fundamental para descrição do estado funcional do paciente internado na UTI, também é importante para direcionar as intervenções e para definição de prognósticos (Samosawala, Vaishali \& Kalyana, 2016). Para realização do diagnóstico da FMA o instrumento frequentemente usado é a avaliação dos seis grupos musculares pela escala MRC (Hermans \& Van den Berghe, 2015; Latronico \& Gosselink, 2015). Porém, além da MRC, outros instrumentos para diagnósticos da FMA foram encontrados nesta revisão e podem ser utilizados.

Samosawala et al. (2016) destacam a importância de métodos objetivos e confiáveis para examinar a FMA na UTI e utilizaram o dinamômetro, que se mostrou confiável e sensível para identificar a redução da força muscular em pacientes críticos, corroborando aos achados de Bragança, Ravetti, Barreto, Ataíde, Carneiro e Teixeira et al. (2019) que viram um desempenho positivo do dinamômetro para avaliar a força muscular quando comparado ao MRC.

A avaliação de força é dificultada pelo uso de sedativos comumente utilizados em UTI fazendo com que os pacientes se encontrem inconscientes e não cooperativos, atrasando o diagnóstico da FMA. O estudo de Xie, Liu, Zheng, Cao, Liu \& Li (2020) utilizou a avaliação da proteína GDF 15, ressaltada como um bom biomarcador para perda muscular e com boa relação associada ao uso do ultrassom a beira leito, sendo uma alternativa a esses pacientes. $\mathrm{O}$ ultrassom também pode ser utilizado independente da cooperação do paciente, sendo um método não invasivo, rápido e de fácil acesso. (Hernández-Socorro, Saavedra, López-Fernández \& Ruiz-Santana, 2018). Kelmenson, Quan e Moss (2018) observaram que a ecogenicidade do ultrassom muscular agrega valor para a previsão do diagnóstico de fraqueza muscular, podendo ser benéfica a utilização rotineira para pacientes críticos. 
O estudo de Patejdl, Walter, Rosener, Sauer, Reuter e Ehler (2019), sugere que achados anormais de ultrassonografia podem apoiar o diagnóstico de FMA na UTI, corroborando com os estudos de Hernández-Socorro et al. (2018) e Kelmenson et al. (2018), especialmente se os achados forem combinados com os níveis séricos de procalcitoninas. $\mathrm{O}$ aumento da ecogenicidade muscular foi relacionado a níveis séricos elevados de proteína syndecan-1, o que reforça a ideia de que processos inflamatórios levam a ecogenicidade muscular anormal.

Kawahara, Suzuki, Yasaka, Nagata, Okamoto e Kita et al. (2016), após medirem a circunferência dos membros inferiores (MMII) e membros superiores (MMSS) de grupos divididos em independentes e dependentes nas atividades de vida diária (AVDs), antes da internação, perceberam que a atrofia muscular foi mais significativa nos MMII do que nos MMSS, em pacientes independentes, durante um breve tempo na UTI.

Peres, Faria, Teixeira e Coelho (2018) observaram que quanto maior o tempo de internação em UTI e de VMI, maior a dificuldade nas AVDs como higiene pessoal, alimentação e principalmente locomoção. Os pacientes apresentaram melhora gradual da independência funcional no decorrer do tempo, porém com 90 dias após alta hospitalar ainda não readquiriram a capacidade funcional apresentada antes da internação.

Com isso, vem se destacando a mobilização precoce para reduzir a fraqueza muscular, na qual os exercícios se iniciam cada vez mais cedo. Atualmente existem diretrizes de exercícios para doentes críticos que são fundamentadas em níveis de mobilidade, que diversificam do exercício passivo a caminhada (França, Gomes, De Lira, Amaral, Vilaça \& Paiva Júnior et al., 2020).

O estudo de França et al. (2020) utilizou o cicloergômetro passivo e estimulação elétrica em pacientes sob ventilação mecânica, onde observaram que essas condutas oferecem contração muscular sem aumentar o esforço cardiovascular, prevenindo assim a atrofia muscular, diminuindo o estresse nitrosativo e aumentando a perfusão dos tecidos, concordando com o estudo de Nakamura, Kihata, Naraba, Kanda, Takahashi e Sonoo et al. (2019) que apresentou que a estimulação elétrica pode impedir a taxa de perda do volume muscular.

Wollersheim, Haas, Wolf, Mai, Spies e Steinhagen-Thiessen et al. (2017) utilizaram a vibração de todo corpo em pacientes críticos como técnica para pacientes que ainda não são capazes de cooperar devido a sedação, os resultados apontaram que a vibração induz a ativação muscular, podendo prevenir a FMA na UTI, e se mostrou segura por não causar alterações hemodinâmicas significativas. Na pesquisa de Sarfati, Moore, Pilorge, Amaru, Mendialdua e Rodet et al. (2018) a inclinação passiva foi usada após cirurgia cardiotorácica em pacientes de UTI, os resultados não demonstraram melhora da força muscular após o uso da técnica, porém sugeriram uma recuperação mais rápida. Ambos artigos sugeriram que as técnicas passivas são eficazes para o tratamento da FMA e não causaram efeitos adversos graves, porém são necessários maior número de estudos para avaliarem essa prática.

A FMA ocorre em pacientes em repouso prolongado e está relacionada a alterações do metabolismo celular decorrente da falta de exercício. A contração muscular ativa é importante no manejo e controle de complicações inerentes em pacientes críticos, Enciso-Olivera, Galvis-Rincón, Torre-Díaz, Devia-León e Camargo-Puerto (2016) embasados no princípio da sobrecarga, utilizaram um protocolo de carga progressiva, objetivando avaliar se as alterações cardiovasculares relacionadas ao exercício limitavam ou impediam a realização de exercício ativo em pacientes críticos, corroborando com o estudo de França et al. (2020), verificaram que a estabilidade nas variáveis de pressão arterial e frequência cardíaca, permitem estabelecer de forma segura o exercício ativo com cargas controladas e monitoradas de forma progressiva. Sendo assim a mobilização precoce vem se tornando rotineira em UTI por se mostrar uma técnica segura e eficaz para prevenir a FMA. 


\section{Conclusão}

A FMA na UTI é uma disfunção frequentemente encontrada em pacientes que permanecem em repouso prolongado no leito, afetando sua qualidade de vida à longo prazo. As ferramentas mais utilizadas para o diagnóstico foram o MRC, a dinamometria e o ultrassom. A mobilização precoce atua na manutenção da força muscular e melhora funcional do doente crítico, com diversas possibilidades de exercícios, sem trazer riscos para o paciente, sendo a terapia de escolha para tratar a FMA na UTI.

\section{Referências}

Aguiar, F. P., Westphal, G. A., Dadam, M. M., Mota, E. C. C., Pfutzenreuter, F., \& França, P. H. C. (2019). Características e preditores de doença crítica crônica na unidade de terapia intensiva. Revista brasileira de terapia intensiva, 31(4), 511-520. https://doi.org/10.5935/0103-507x.20190088

Ballve, L. P. D, Dargains, N., Inchaustegui, J. G. U., Bratos, A., Percaz, M. M., Ardariz, C. B., Cagide, S., Balestrieri, C., Gamarra, C., Paz, D., Rotela, E., Muller, S., Bustos, F., Castro, R. A., \& Settembrino, E. (2017). Weakness acquired in the intensive care unit. Incidence, risk factors and their association with inspiratory weakness. Observational cohort study. Revista Brasileira de Terapia Intensiva, 29(4), 466-475. https://doi.org/10.5935/0103-507x.20170063

Bragança, R. D., Ravetti C. G., Barreto, L., Ataíde, T. B. L. S., Carneiro, R. M., Teixeira, A. L., \& Nobre, V. (2019). Use of handgrip dynamometry for diagnosis and prognosis assessment of intensive care unit acquired weakness: A prospective study. Heart \& Lung, 48(6), 532-537. https://doi.org/10.1016/j.hrtlng.2019.07.001

Castro-Avila, A. C., Serón, P., Fan, E., Gaete, M., \& Mickan, S. (2015). Effect of Early Rehabilitation during Intensive Care Unit Stay on Functional Status: Systematic Review and Meta-Analysis. PloS one, 10(7), e0130722. https://doi.org/10.1371/journal.pone.0130722

Doiron, K. A., Hoffmann, T. C., \& Beller, E. M. (2018). Early intervention (mobilization or active exercise) for critically ill adults in the intensive care unit. The Cochrane database of systematic reviews, 3(3), CD010754. https://doi.org/10.1002/14651858.CD010754.pub2

Enciso-Olivera, C. O., Galvis- Rincón, J. C., Torre-Díaz, E., Devia-León, A., \& Camargo-Puerto, D. A. (2016). Efectos cardiovasculares de un protocolo de reacondicionamiento físico en pacientes críticos de tres centros asistenciales en Bogotá, Colombia. Medicas UIS, 29(2), 161-173. http://dx.doi.org/10.18273/revmed.v29n2-2016014.

França, E. E. T., Gomes, J. P. V., De Lira, J. M. B., Amaral, T. C. N., Vilaça, A. F., Paiva Júnior, M. D. S., Elihimas Júnior, U. F., Correia Júnior, M. A. V., Forgiarini Júnior, L. A., Costa, M. J. C., Andrade, M. A., Ribeiro, L. C., \& De Castro, C. M. M. B. (2020). Acute effect of passive cycle-ergometry and functional electrical stimulation on nitrosative stress and inflammatory cytokines in mechanically ventilated critically ill patients: a randomized controlled trial. Revista Brasileira de Pesquisa Médica e Biológica, 53(4), e8770. https://doi.org/10.1590/1414-431x20208770

Hermans, G., \& Van den Berghe, G. (2015). Clinical review: intensive care unit acquired weakness. Critical care (London, England), 19(1), 274. https://doi.org/10.1186/s13054-015-0993-7

Hernandéz-Socorro, C. R., Saavedra, P., López-Fernández, J. C., \& Ruiz-Santana S. (2018). Assessment of Muscle Wasting in Long-Stay ICU Patients Using a New Ultrasound Protocol. Nutrients, 10(12), 1849. https://doi.org/10.3390/nu10121849

Hodgson, C. L., \& Tipping, C. J. (2017). Physiotherapy management of intensive care unit-acquired weakness. Journal of Physiotherapy, 63(1), 4-10. https://doi.org/10.1016/j.jphys.2016.10.011

Kawahara, K., Suzuki, T., Yasaka, T., Nagata, H., Okamoto, Y., Kita, K., \& Morisaki, H. (2016). Evaluation of the site specificity of acute disuse muscle atrophy developed during a relatively short period in critically ill patients according to the activities of daily living level: A prospective observational study. Australian Critical Care, 30(1), 29-36. https://doi.org/10.1016/j.aucc.2016.01.003

Kelmenson, D. A., Quan, D., \& Moss, M. (2018). What is the diagnostic accuracy of single nerve conduction studies and muscle ultrasound to identify critical illness polyneuromyopathy: a prospective cohort study. Critical Care, 22(1), 342. https://doi.org/10.1186/s13054-018-2281-9

Latronico, N., \& Gosselink, R. (2015). Abordagem dirigida para o diagnóstico de fraqueza muscular grave na unidade de terapia intensiva. Revista Brasileira de Terapia Intensiva, 27(3), 199-201. https://doi.org/10.5935/0103-507X.20150036

Nakamura, K., Kihata, A., Naraba, H., Kanda, N., Takahashi, Y., Sonoo, T., Hashimoto, H., \& Morimura, N. (2019). Efficacy of belt electrode skeletal muscle electrical stimulation on reducing the rate of muscle volume loss in critically ill patients: A randomized controlled trial. Journal of Rehabilitation Medicine, 51(9), 705-711. doi: 10.2340/16501977-2594

Patejdl, R., Walter, U., Rosener, S., Sauer, M., Reuter, D. A., \& Ehler, J. (2019). Muscular Ultrasound, Syndecan-1 and Procalcitonin Serum Levels to Assess Intensive Care Unit-Acquired Weakness. Canadian Journal of Neurological Sciences, 46(2), 234-242. doi:10.1017/cjn.2018.390

Peres, N. T., Faria, I. D., Teixeira, A. P. A., \& Coelho, R. R. (2018). Avaliação da independência funcional em pacientes críticos até 90 dias após alta da UTI. Fisioterapia Brasil, 19(2), 162-170. avaliacao-da-independencia-funcional-em-pacientes-criticos-ate-_UZXfN1c.pdf (bvsalud.org)

Rother, E. T. (2007). Revisão Sistemática X Revisão Narrativa. Acta Paulista de Enfermagem, 20(2)vi. https://www.scielo.br/pdf/ape/v20n2/a01v20n2.pdf

Sachetti, A., Dal'Acqua, A. M., Lemos, F. A., Naue, W. S., Santos, L. J., Bianchi, T., \& Dias, A. S. (2017). Efeitos da estimulação elétrica neuromuscular sobre a mobilidade diafragmática de pacientes críticos: ensaio clínico randomizado. ConScientiae Saúde, 16(2), 224-233. DOI:10.5585 
Research, Society and Development, v. 10, n. 8, e12010817077, 2021

(CC BY 4.0) | ISSN 2525-3409 | DOI: http://dx.doi.org/10.33448/rsd-v10i8.17077

Samosawala, N. R., Vaishali, K., \& Kalyana B. C. (2016). Measurement of muscle strength with handheld dynamometer in Intensive Care Unit. Indian Journal of Critical Care Medicine, 20(1): 21-6. DOI: 10.4103/0972-5229.173683

Sarfati, C., Moore, A., Pilorge, C., Amaru, P., Mendialdua, P., Rodet, E., Stephan, F., \& Rezaiguia-Delclaux, S. (2018). Efficacy of early passive tilting in minimizing ICU-acquired weakness: A randomized controlled trial. Journal of Critical Care, 46, 37-43. https://doi.org/10.1016/j.jcrc.2018.03.031

Sommers, J., Wieferink, D. C., Dongelmans, D. A., Nollet, F., Engelbert, R. H. H., \& van der Schaaf, M. (2017). Body weight-supported bedside treadmill training facilitates ambulation in ICU patients: An interventional proof of concept study. Journal of Critical Care, 41, 150-155. https://doi.org/10.1016/j.jcrc.2017.05.010

Veldema, J., Bösl, K., Kugler, P., Ponfick, M., Gdynia, H. J., \& Nowak, D. A. (2019). Cycle ergometer training vs resistance training in ICU-acquired weakness. Acta Neurologica Scandinavica, 140(1), 62-71. https://doi.org/10.1111/ane.13102

Vicente, A., Amoza, L. R., García, C. R., Tocalini, P., Prieto, L., Savio, P., Simioni, B. M., Ferrario, A., Cura J. A., Tozzi, A. W., Villarruel, M., Verde, A. G., Garegnani, I. L., \& Virgilio, A. S. (2020). Epidemiological Characteristics and Risk Factors of Adult Patients with Intensive Care Unit-Acquired Weakness. Revista Americana de Medicina Respiratoria, 20(3), 225-234. http://www.ramr.org/articulos/volumen_20_numero_3/articulos_originales/articul os_originales_epidemiological_characteristics_and_risk_factors_of_adult_patients_with_intensive_care.pdf

von Elm E., Altman D. G., Egger M., Pocock S. J., Gøtzsche P. C., Vandenbroucke J. P \& STROBE Initiative. (2008). The Strengthening the Reporting of Observational Studies in Epidemiology (STROBE) statement: guidelines for reporting observational studies. Journal of Clinical Epidemiology, 61(4):344-9. doi: 10.1016/j.jclinepi.2007.11.008.

Witteveen E., Wieske L., Sommers J., Spijkstra J. J., de Waard M. C., Endeman H., Rijkenberg S., de Ruijter W., Sleeswijk M., Verhamme C., Schultz M. J., van Schaik I. N. \& Horn J. (2020). Early Prediction of Intensive Care Unit-Acquired Weakness: A Multicenter External Validation Study. Journal of Intensive Care Medicine, 35(6), 595-605. doi: 10.1177/0885066618771001.

Wollersheim, T., Haas, K., Wolf, S. Mai, K., Spies, C., Steinhagen-Thiessen, E., Wernecke, K. D., Spranger, J., \& Weber-Carstens, S. (2017). Whole-body vibration to prevent intensive care unit-acquired weakness: safety, feasibility, and metabolic response. Critical Care, 21(1), 9. https://doi.org/10.1186/s13054016-1576-y

Xie, Y., Liu, S., Zheng, H., Cao, L., Liu, K., \& Li, X. (2020). Utility of Plasma GDF-15 for Diagnosis and Prognosis Assessment of ICU-Acquired Weakness in Mechanically Ventilated Patients: Prospective Observational Study. BioMed Research International, 3630568. https://doi.org/10.1155/2020/3630568

Zhou, W., Shi, B., Fan, Y., \& Zhu, J. (2020). Effect of early activity combined with early nutrition on acquired weakness in ICU patients. Medicine, 99(29), e21282. https://doi.org/10.1097/MD.0000000000021282 